\title{
Chiral separation of hydroxyflavanones in cyclodextrin-modified capillary zone electrophoresis using sulfated cyclodextrins as chiral selectors
}

\author{
Chen-Hsing Lin ${ }^{a}$, Wei-Ru Fang ${ }^{b}$, Chia-Ming Kuo ${ }^{b}$, Wing-Yao Chang ${ }^{b}$, \\ Yu-Chih Liu ${ }^{\mathrm{a}}$, Wann-Yin Lin ${ }^{\mathrm{b}}$, Jong-Chang Wu ${ }^{\mathrm{a}}$, Ching-Erh Lin ${ }^{\mathrm{a}} \mathrm{b}, *, 1$ \\ ${ }^{a}$ Department of Applied Chemistry, Fooyin University, Ta-Liao, Kaohsiung County 831, Taiwan \\ ${ }^{\mathrm{b}}$ Department of Chemistry, National Taiwan University, Taipei 10671, Taiwan
}

Received 20 December 2007; received in revised form 22 January 2008; accepted 21 February 2008

Available online 29 February 2008

\begin{abstract}
Chiral separations of three hydroxyflavanone aglycones, including $2^{\prime}-$, $3^{\prime}$-, and 4'-hydroxyflavanone, in capillary zone electrophoresis (CZE) using randomly sulfate-substituted $\beta$-cyclodextrin $(\mathrm{S}-\beta-\mathrm{CD})$ or dual cyclodextrin $(\mathrm{CD})$ systems consisting of S- $\beta-\mathrm{CD}$ and a neutral $\mathrm{CD}$ at low pH were investigated. The results indicate that $\mathrm{S}-\beta-\mathrm{CD}$ is an excellent chiral selector for enantioseparation of $2^{\prime}$-hydroxyflavanone and is a good chiral selector for $3^{\prime}$-hydroxyflavanone. Depending on the concentration of S- $\beta$-CD ranging from 2.0 to $0.75 \%$ (w/v), the enantioresolution values were 10.5-19.5 and 1.8-3.4 for $2^{\prime}$ - and $3^{\prime}$-hydroxyflavanone, respectively. The enantiomers of 4 '-hydroxyflavanone could be effectively separated with S- $\beta-C D$ at a concentration of $2.0 \%$ (w/v) within $20 \mathrm{~min}$. The enantioselectivity and enantioresolution follow the order $2^{\prime}$-hydroxyflavanone $\gg 3^{\prime}-$ hydroxyflavanone $>4$ '-hydroxyflavanone. Alternatively, with the addition of sodium dodecyl sulfate (SDS) monomers at low concentrations in the electrophoretic system, enantioselectivity of these hydroxyflavanone aglycones could be enhanced with dual CD systems. In this case, SDS monomer acted as a complexing agent probably first with $S-\beta-C D$ and then subsequently with the analytes for increasing the effective electrophoretic mobility of the analytes towards the anode and as a selectivity controller for affecting the selectivity of hydroxyflavanones. Better enantioseparation between 2 -hydroxyflavanone and 3'-hydroxyflavanone could be achieved with a dual CD system consisting of S- $\beta-C D$ and $\gamma$-CD than that with S- $\beta$-CD and $\beta$-CD. In addition, possible chiral recognition mechanisms of hydroxyflavanones are discussed.
\end{abstract}

(C) 2008 Elsevier B.V. All rights reserved.

Keywords: Chiral separation; Enantioseparation; Capillary electrophoresis; Sulfated $\beta$-cyclodextrin; Flavonoid; Flavanone; Hydroxyflavanone

\section{Introduction}

Flavonoids, characterized by a common 2-phenyl-benzopyran-4-one basic structure, constitute one of the largest group of naturally occurring compounds [1]. Flavanone, one of the twelve subclasses of flavonoids, is predominantly present in citrus fruits and vegetables where they are usually found as $7-O$-glycosides. Flavanone aglycones possess a stereogenic center in $\mathrm{C}_{2}$ position. Thus flavanone aglycones exist as pairs of enantiomers, whereas flavanone-7- $O$-glycosides possess an additional D-configurated mono- or disaccharide moiety and accordingly exist as pairs of

\footnotetext{
* Corresponding author: Department of Chemistry, National Taiwan University, Taipei 10671, Taiwan. Tel.: +8862 33661158; fax: +886223636359.

E-mail address: celin@ntu.edu.tw (C.-E. Lin).

${ }^{1}$ Present address: Department of Applied Chemistry, Fooyin University, Ta-Liao, Kaohsiung County 831, Taiwan. Tel.:+886 7 7811151x667; fax: +886 7 7826732; E-mail:sc030@ mail.fy.edu.tw.
}

epimers. Flavanone aglycones can be converted from flavanone7-O-glycosides by hydrolysis.

Flavonoids are of biological and physiological importance, due to their antioxidant capacities [2] and a wide range of antimicrobial and pharmaceutical activities [3,4]. They are used as anti-inflammatory, antiviral, antiallergic, antibiotic, and anticarcinogenic compounds [3,5]. They also exhibit inhibitory effects for a number of enzymes, including kinases, transferases, hydrolases, and ATPases [5,6]. In nature, in some cases, only one of the enantiomers of flavonoids, such as (-)$2 \mathrm{~S}$-naringenin $\left(4^{\prime}, 5,7\right.$-trihydroxyflavanone), is formed in the enzymatical pathway [4]. As for some pharmaceutical compounds, pharmaceutical effects of flavonoids may be resulted from a single enantiomer. In some cases, adverse effects may even be resulted from the opposite antipode [6]. The expanding interest in flavonoids in the pharmaceutical and biomedical sciences will certainly lead to an increased need for enantioseparation of flavonoids. Thus the development of 
analytical methods for enantioseparation of flavonoids is desirable.

Applications of capillary electrophoresis (CE) technique to the separation and/or analysis of flavonoids have previously been reported for the past decades [7-20]. However, papers dealing with chiral separation of flavonoids are quite few [21-29], especially on the enantioseparation of flavanone aglycones [23-26]. It was found that the enantiomers of flavanone aglycones (hesperetin and naringenin) could only be baseline resolved with SBE- $\beta-\mathrm{CD}$ at high concentrations $(>40 \mathrm{mg} / \mathrm{mL})$ and that baseline resolution was not obtained by $\mathrm{CE}$ for the enantiomers of $2^{\prime}$-hydroxyflavanone using SBE$\beta-C D$ as a chiral selector [23]. Recently, the stereoisomeric separations of selected flavanones (nargingenin, hesperetin, eriodictyol, pinostrobin, isosakuranetin, homoeriodictyol, and 4 -hydroxyflavanone) have been performed by CZE using neutral CD derivatives (HP- $\gamma-C D$, methyl- $\beta-C D$, methyl- $\gamma-C D$ ) and charged CDs (carboxylmethyl- $\beta-C D$, carboxymethyl- $\gamma$ $\mathrm{CD}$, and sulfo- $\beta-\mathrm{CD}$ ) as chiral selectors in a phosphate/borate buffer at neutral or alkaline $\mathrm{pH}(9-11)$ [24]. In this study, the enantiomeric separation of $4^{\prime}$-hydroxyflavanone was achieved with the best resolution of 1.39 with sulfo- $\beta-\mathrm{CD}$ [24].

On the other hand, micellar electrokinetic chromatography (MEKC) has been applied for enantioseparation of some flavanone aglycones with the addition of $\gamma$-CD in a background electrolyte containing SDS micelles at $\mathrm{pH}$ 8.3. However, the enantioresolution (Rs) values for these analytes were in the range of $0.69-1.16$ [25]. $\delta$-CD was employed as a chiral selector for enantioseparation of some flavanone glycosides in MEKC with SDS micelles at pH 9 [26]. Complete enantioseparation of naringin, neohesparidin and neoeriocitrin was achieved with $\delta$-CD as a chiral selector. Very recently, a review article on methods of analysis and separation of chiral flavonoids was reported [27].

In recent years, the use of sulfate-substituted CDs and the use of dual CD systems as chiral selectors have become attractive in chiral CE separation [30-41]. In this report, we present the results on enantioseparations of hydroxyflavanones either with randomly sulfate-substituted $\beta-C D(S-\beta-C D)$ alone or with dual CD systems consisting of $S-\beta-C D$ and a neutral $C D$ as chiral selectors in a phosphate buffer at $\mathrm{pH} 3.0$ with or without the addition of SDS monomers at low concentrations. The influences of the addition of SDS monomers, which acted as a complexing agent for increasing the effective electrophoretic mobility of the analytes towards the anode and as a selectivity controller for affecting the selectivity of hydroxyflavanones on the separation of hydroxyflavanones were also studied. Moreover, in correlation with the molecular structures of hydroxyflavanones, possible chiral recognition mechanisms are discussed.

\section{Experimental}

\subsection{Apparatus}

All CE separations were performed on a Beckman P/ACE System MDQ equipped with a photodiode array detector for absorbance measurements at 214 nm (Beckman Coulter, Fullerton, CA, USA). Uncoated fused-silica capillaries purchased from Polymicro Technologies (Phoenix, AZ, USA) were used. The dimensions of the capillary were $60.2 \mathrm{~cm} \times 50 \mu \mathrm{m}$ i.d. The effective length of the capillary was $50 \mathrm{~cm}$ from the injection end of the capillary. The CE system was interfaced with a microcomputer. System Gold software of Beckman was used for data acquisition. For $\mathrm{pH}$ measurements, a pH meter (Suntex Model SP-701, Taipei, Taiwan) was employed with a precision of \pm 0.01 $\mathrm{pH}$ unit.

\subsection{Chemicals and reagents}

All three hydroxyflavanone racemates were obtained from ICC (New York, NY, USA). Randomly sulfate-substituted $\beta-C D$ (S- $\beta-C D)$ was purchased from Sigma-Aldrich (St. Louis, MO, USA). $\beta-C D$ and SDS were supplied from Merck (Darmstadt, Germany). $\gamma$-CD was obtained from TCI (Tokyo, Japan). All other chemicals were of analytical grade. Deionized water was prepared with a Milli-Q system (Millipore, Bedford, MA. USA).

Standard solutions of the analytes, at a concentration of $30 \mu \mathrm{g} / \mathrm{mL}$ were prepared by dissolving analytes in an aqueous solution containing $4.5 \%$ acetonitrile. The $\mathrm{pH}$ of a phosphate buffer was adjusted to 3.0 by adding a proportion of $50 \mathrm{mM}$ sodium dihydrogenphosphate solution to the same concentration of phosphoric acid solution until the $\mathrm{pH}$ meter read 3.0. All buffer solutions, freshly prepared weekly and stored in a refrigerator before use, were filtered through a membrane filter $(0.22 \mu \mathrm{m})$.

\subsection{Electrophoretic procedure}

When a new capillary was used, the capillary was washed 30 min with $1.0 \mathrm{M} \mathrm{NaOH}$ solution, followed by $30 \mathrm{~min}$ with deionized water at $25^{\circ} \mathrm{C}$. The washing procedure was repeatedly carried out at least three times. Before each injection, the capillary was washed for 5 min with running buffer and postwashed for $3 \mathrm{~min}$ with deionized water, $5 \mathrm{~min}$ with $0.1 \mathrm{M} \mathrm{NaOH}$, and then 5 min again with deionized water to maintain proper reproducibility of run-to-run injections. Sample injections were done in a hydrodynamic mode over $6 \mathrm{~s}$ under a pressure of $1.0 \mathrm{psi}$ at $25^{\circ} \mathrm{C}$. The measurements were run at least in triplicate to ensure reproducibility. An applied voltage of $-21 \mathrm{kV}$ in the reversed polarity mode for phosphate buffer was selected to keep the total current less than $100 \mu \mathrm{A}$. The detection wavelength was set at $214 \mathrm{~nm}$ (pH 3.0). Peak identification was conducted by spiking with the analyte to be identified. Mesityl oxide was used as neutral marker. At pH 3.0, the electro-osmotic mobility is very small and thus it took too long to measure its magnitude.

\subsection{Mobility calculations}

The apparent mobility of analytes was calculated from the observed migration times with the equation:

$\mu_{\mathrm{app}}=\frac{L_{\mathrm{d}} L_{\mathrm{t}}}{V t_{\mathrm{m}}}$ 
where $\mu_{\text {app }}$ is the apparent mobility, $t_{\mathrm{m}}$ is the migration time measured directly from the electropherogram, $L_{\mathrm{t}}$ is the total length of capillary, $L_{\mathrm{d}}$ is the length of capillary between injection and detection, and $V$ is the applied voltage. The enantioselectivity $(\alpha)$ of basic analytes was calculated with the equation:

$\alpha=\frac{\mu_{\mathrm{app}, \mathrm{b}}}{\mu_{\mathrm{app}, \mathrm{a}}}$

where the subscripts $a$ and $b$ refer to the first and second enantiomer, respectively.

\section{Results and discussion}

In chiral $\mathrm{CE}$, buffer $\mathrm{pH}$ and the concentration of chiral selector are the two most important separation parameters which need to be optimized. In this study, CE experiments were performed in the reversed-polarity using S- $\beta-C D$ as chiral selectors. Thus, the electro-osmotic mobility was suppressed to zero or nearly zero in order to minimize the migration time of the analytes. Therefore, it is appropriate to keep buffer $\mathrm{pH}$ at 3.0.

\section{1. $S-\beta-C D$ as a chiral selector}

Fig. 1 shows the structures of the three hydroxyflavanones studied. As the $\mathrm{p} K_{\mathrm{a}}$ values of $2^{\prime}$ - and $4^{\prime}$-hydroxyflavanones were determined to be 7.54 and 8.00 , respectively, hydroxyflavanones are expected to be in the neutral form at $\mathrm{pH}$ 3.0. Thus by complexation with $S-\beta-C D$, the analytes migrate towards the anode in the reversed direction of the suppressed electro-osmotic flow. As $S-\beta-C D$ is composed of a number of sulfated $\beta-C D$ (typically with substitution $7-11 \mathrm{~mol} / \mathrm{mol} \beta-\mathrm{CD})$, the concentration of $\mathrm{S}-\beta-\mathrm{CD}$ is given in $\%(\mathrm{w} / \mathrm{v})$, instead of $\mathrm{mM}$. Fig. 2 shows the electropherogram of hydroxyflavanones obtained with the addition of $2.0 \%(\mathrm{w} / \mathrm{v}) \mathrm{S}-\beta-\mathrm{CD}$ in a phosphate buffer at $\mathrm{pH}$ 3.0. As can be seen, the enantiomers of the three hydroxyflavanone aglycones could be effectively separated using S- $\beta-C D$<smiles>O=C1C[C+](c2ccccc2O)Oc2ccccc21</smiles>

2'-Hydroxyflavanone<smiles>O=C1C[C+](c2cccc(O)c2)Oc2ccccc21</smiles>

3'-Hydroxyflavanone<smiles>O=C1C[C@H](c2ccc(O)cc2)Oc2ccccc21</smiles>

\section{4'-Hydroxyflavanone}

(B)

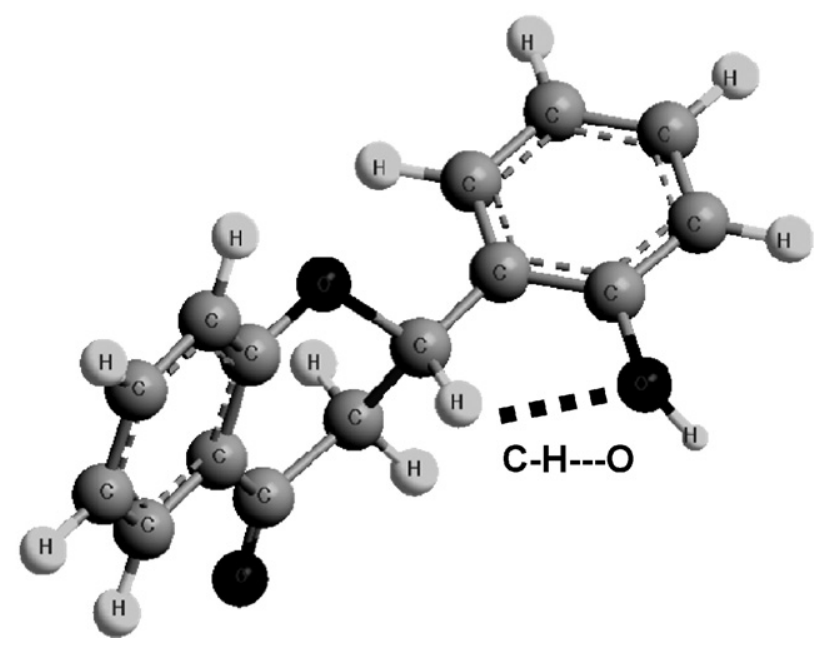

Fig. 1. Molecular structures of hydroxyflavanones: (A) schematic structures, (B) enantiomeric structure of $2^{\prime}$-hydroxyflavanone indicating the presence of $\mathrm{C}-\mathrm{H} \cdots \mathrm{O}$ intramolecular hydrogen bonding. 


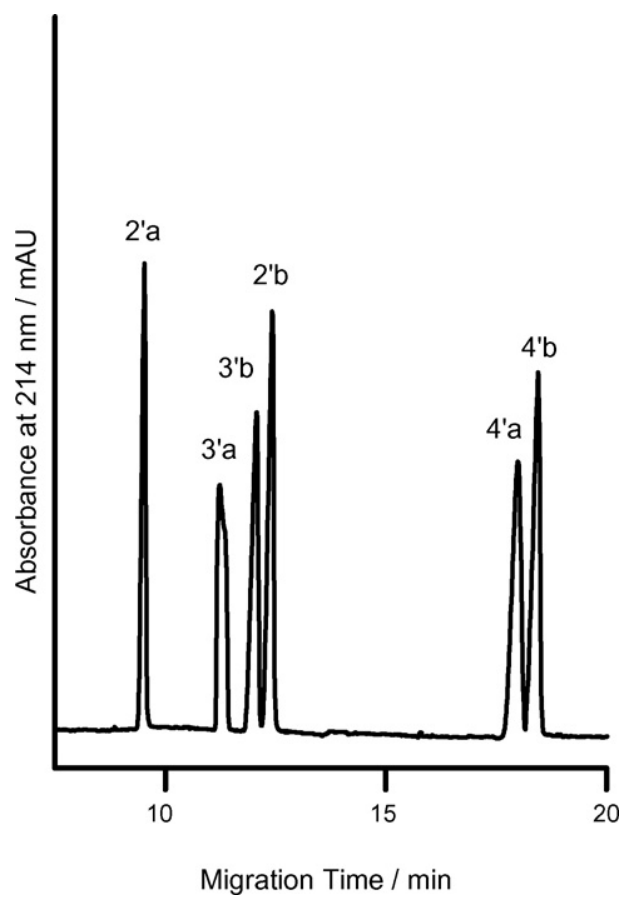

Fig. 2. Electropherogram of hydroxyflavanones obtained with $2.0 \%$ (w/v) S- $\beta$ $\mathrm{CD}$ in a phosphate buffer $(50 \mathrm{mM})$ at $\mathrm{pH} 3.0$. Capillary, $50.2 \mathrm{~cm} \times 50 \mu \mathrm{m}$, i.d. Sample concentration, $30 \mu \mathrm{g} / \mathrm{mL}$, detection wavelength, $214 \mathrm{~nm}$. Other operating conditions, $-21 \mathrm{kV}, 25^{\circ} \mathrm{C}$. Peak identification, $2^{\prime}=2^{\prime}$-hydroxyflavanone, $3^{\prime}=3^{\prime}$-hydroxyflavanone, $4^{\prime}=4^{\prime}$-hydroxyflavanone.

as a chiral selector at a concentration around $2.0 \%(\mathrm{w} / \mathrm{v})$. The first enantiomeric peak of $2^{\prime}$-hydroxyflavanone eluted before the two enantiomeric peaks of $3^{\prime}$-hydroxyflavanone, then followed by the second enantiomeric peak of $2^{\prime}$-hydroxyflavanone. The two enantiomeric peaks of $4^{\prime}$-hydroxyflavanone appeared last in the electropherograms. The enantiomers of $4^{\prime}$-hydroxyflavanone could be effectively separated with S- $\beta$-CD within $20 \mathrm{~min}$. The enantioselectivity follows the order $2^{\prime}$-hydroxyflavanone $\gg 3^{\prime}$ hydroxyflavanone $>4^{\prime}$-hydroxyflavanone. The enantiomers of $4^{\prime}$-hydroxyflavanone could not be detected within $30 \mathrm{~min}$ when using S- $\beta-C D$ at a concentration lower than $1.0 \%(\mathrm{w} / \mathrm{v})($ not shown). On the other hand, the use of S- $\beta-C D$ at a concentration greater than $3.0 \%(\mathrm{w} / \mathrm{v})$ was not suitable because the migration window became too narrow and the two enantiomeric peaks of $4^{\prime}$-hydroxyflavanone, as well as the second enantiomeric peak of $3^{\prime}$-hydroxyflavanone could not be completely separated from the second enantiomeric peak of $2^{\prime}$-hydroxyflavanone.

The enantioresolution (Rs) of the two enantiomeric peaks, defined as the peak separation divided by the average of the widths, and the enantioselectivity $(\alpha)$ increased with decreasing the concentration of S- $\beta-C D$. Table 1 illustrates the effect of $S-\beta-C D$ concentration on the enantioresolution and enantioselectivity of hydroxyflavanones. The Rs values increased from 10.5 to 19.5 and from 1.8 to 3.4 for $2^{\prime}$ - and $3^{\prime}$-hydroxyflavanone, respectively, when the concentration of S- $\beta-C D$ decreased from 2.0 to $0.75 \%(\mathrm{w} / \mathrm{v})$. As demonstrated, $\mathrm{S}-\beta-\mathrm{CD}$ is an excellent chiral selector for enantioseparation of $2^{\prime}$-hydroxyflavanone and is an effective chiral selector for enantioseparation of $3^{\prime}$ hydroxyflavanone in reversed mode at low $\mathrm{pH}$.

It is well established that the extent of the variation of the mobility of an analyte as a function of the concentration of a chiral selector, which depends actually on both the binding constant and limiting electrophoretic mobility, may reflect the binding strength of an analyte with the chiral selector [42]. As the extents of the variations of the mobility of the three hydroxyflavanones studied are about the same, it is expected that the interactions of these three hydroxyflavanones with S$\beta-C D$ may vary only to a small extent and that the migration order of hydroxyflavanones depends primarily on the limiting electrophoretic mobility, which can be determined from the mobility of hydroxyflavanones in the dissociated form at a $\mathrm{pH}$ above $\mathrm{p} K_{\mathrm{a}}$ plus two $\mathrm{pH}$ units. In fact, the limiting electrophoretic mobility of $2^{\prime}$ - and $4^{\prime}$-hydroxyflavanone determined are $-1.58 \times 10^{-4}$ and $-1.01 \times 10^{-4} \mathrm{~cm}^{2} \mathrm{~V}^{-1} \mathrm{~s}^{-1}$, respectively. This is agreeable with the migration order of hydroxyflavanones observed, i.e., 2'-hydroxyflavanone $<3^{\prime}$-hydroxyflvanone $<4^{\prime}$ hydroxyflavanone.

\subsection{1. $S-\beta-C D$ with SDS monomers as complexing agent and selectivity controller}

It has been shown that $\beta$-CD interacts strongly with SDS micelles, and with SDS monomers as well [43-45]. Consequently, enantioseparation of analytes in $\mathrm{CE}$ with $\beta-\mathrm{CD}$ as the chiral selector can be significantly affected by the presence of SDS surfactant [46]. As S- $\beta-C D$ is also expected to interact with SDS monomers to a certain extent, it is of interest to examine the effect of SDS monomer as a complexing agent probably first with $S-\beta-C D$ and then subsequently with the analytes for increasing the electrophoretic mobility of hydroxyflavanones and as a selectivity controller for affecting the selectivity of hydroxyflavanones. Fig. 3 shows the electropherogram of hydroxyflavanones obtained using S- $\beta-\mathrm{CD}(2.0 \%(\mathrm{w} / \mathrm{v}))$ with the addition of $2.0 \mathrm{mM}$ SDS monomers in a phosphate buffer at $\mathrm{pH}$ 3.0. The electrophoretic mobility of hydroxyflavanones, especially $4^{\prime}$-hydroxyflavanone was found to increase

Table 1

Effect of S- $\beta$-CD concentration on the enantioresolution and enantioselectivity of hydroxyflavanones at $\mathrm{pH} 3.0$

\begin{tabular}{llll}
\hline Analytes & S- $\beta$-CD concentration $(\mathrm{w} / \mathrm{v}, \%)$ & Enantioresolution $(\mathrm{Rs})$ & Enantioselectivity $(\alpha)$ \\
\hline 2'-Hydroxyflavanone & 2.0 & 10.5 & 1.3 \\
& 0.75 & 19.5 & 1.68 \\
3'-Hydroxyflavanone & 2.0 & 1.8 & 1.07 \\
4'-Hydroxyflavanone & 0.75 & 3.4 & 1.10 \\
\hline
\end{tabular}




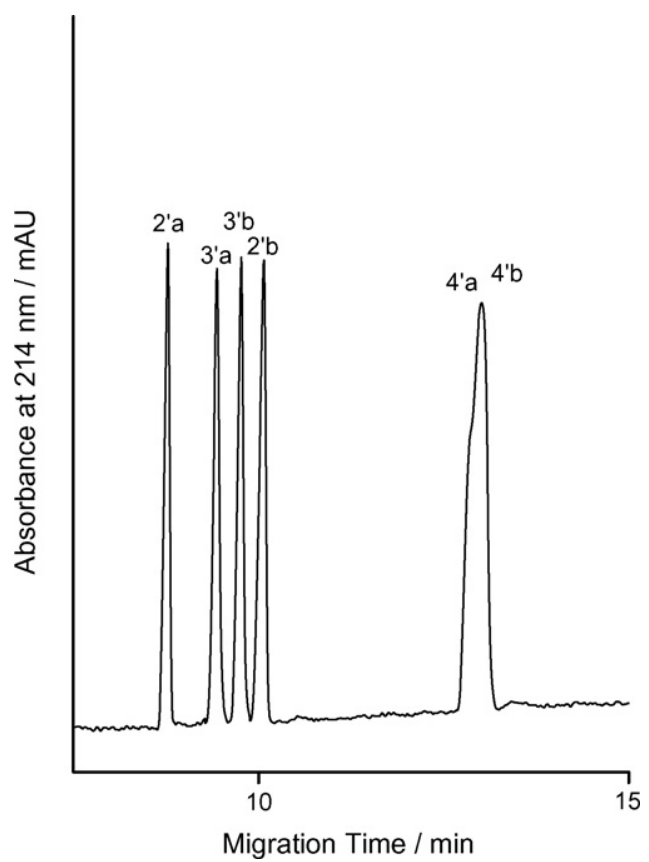

Fig. 3. Electropherograms of hydroxyflavanones obtained with $2.0 \%$ (w/v) S$\beta$-CD with the addition of $2 \mathrm{mM}$ SDS monomers in a phosphate buffer $(50 \mathrm{mM})$ at $\mathrm{pH}$ 3.0. Other operating conditions are the same as for Fig. 2.

considerably and consequently the selectivity of hydroxyflavanones was significantly affected with the addition of SDS monomers. As a matter of fact, 4'-hydroxyflavanone could be eluted within $15 \mathrm{~min}$, but with diminished enantioresolution.

\subsection{Dual CD systems for enantioseparation}

\subsection{1. $S-\beta-C D /$ neutral $C D$ as chiral selectors}

It has been shown that dual $\mathrm{CD}$ systems consisting of a charged $C D$ and a neutral $C D$, such as $\beta-C D$ or $\gamma-C D$, as chiral selectors are often advantageously employed for enantioseparations of chiral compounds [33-41]. It is expected that the mobility of hydroxyflavanones (towards the anode) decreases with increasing the concentration of a neutral $\mathrm{CD}$ at a given concentration of S- $\beta-C D$ in a dual CD system. Consequently, the migration time of hydroxyflavanones increases to a certain extent. With the addition of $2 \mathrm{mM} \beta-\mathrm{CD}$ in the presence of $2.0 \%$ $(\mathrm{w} / \mathrm{v}) \mathrm{S}-\beta-\mathrm{CD}$ in a phosphate buffer at $\mathrm{pH} 3.0$, the signals of $4^{\prime}$-hydroxyflavanone could not appear in the electropherogram within $30 \mathrm{~min}$.

With a dual CD system consisting of S- $\beta-C D$ and $\gamma-C D$, the electrophoretic mobility of hydroxyflavanones decreased to a considerably less extent than that with a dual CD system consisting of $S-\beta-C D$ and $\beta-C D$. This is because the interaction of hydroxyflavanone with $\gamma$-CD is much weaker than that with $\beta-C D$. With the addition of $\gamma-C D$ at concentrations less than $6.0 \mathrm{mM}, 4^{\prime}$-hydroxyflavanone could be eluted within $30 \mathrm{~min}$. It is noted that the enantioresolution of $2^{\prime}$-hydroxyflavanone increases significantly by increasing the concentration of $\gamma$ $\mathrm{CD}$. The two enantiomeric peaks of $3^{\prime}$-hydroxyflavanone were completely resolved and were well separated from the second enantiomeric peak of $2^{\prime}$-hydroxyflavanone. However, the two enantiomers of $4^{\prime}$-hydroxyflavanone were unable to resolve completely.

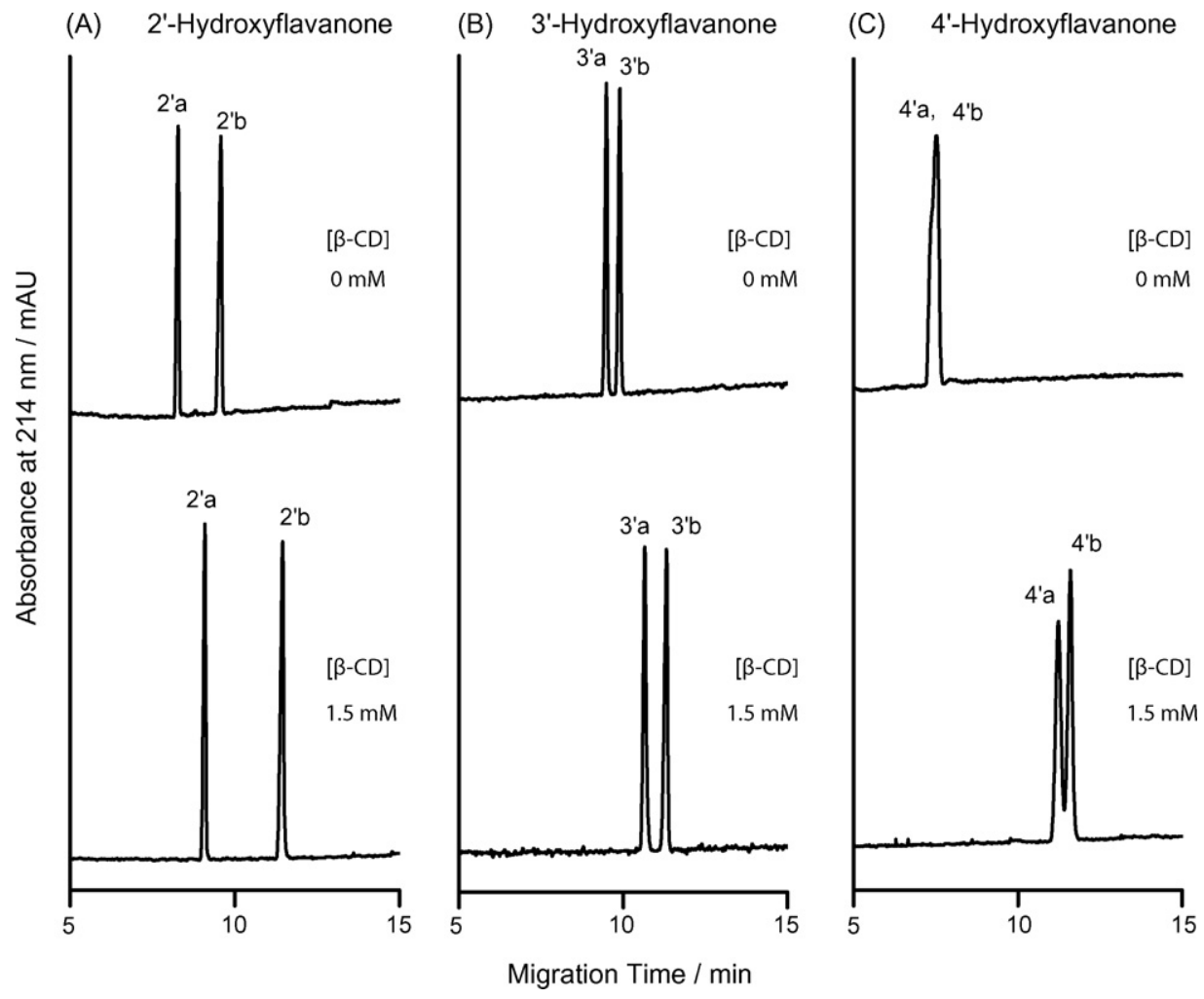

Fig. 4. Electropherogram of hydroxyflavanones obtained with a dual CD systems composed of $2.0 \%(\mathrm{w} / \mathrm{v}) \mathrm{S}-\beta-\mathrm{CD}$ and $1.5 \mathrm{mM} \beta-\mathrm{CD}$ with the addition of $2.0 \mathrm{mM}$ SDS monomers in a phosphate buffer $(50 \mathrm{mM})$ at pH 3.0, together with a CD system without the addition of $\beta$-CD: (A) $2^{\prime}$-hydroxyflavanone, (B) $3^{\prime}$-hydroxyflavanone, and (C) $4^{\prime}$-hydroxyflavanone. Other operating conditions are the same as for Fig. 2. 


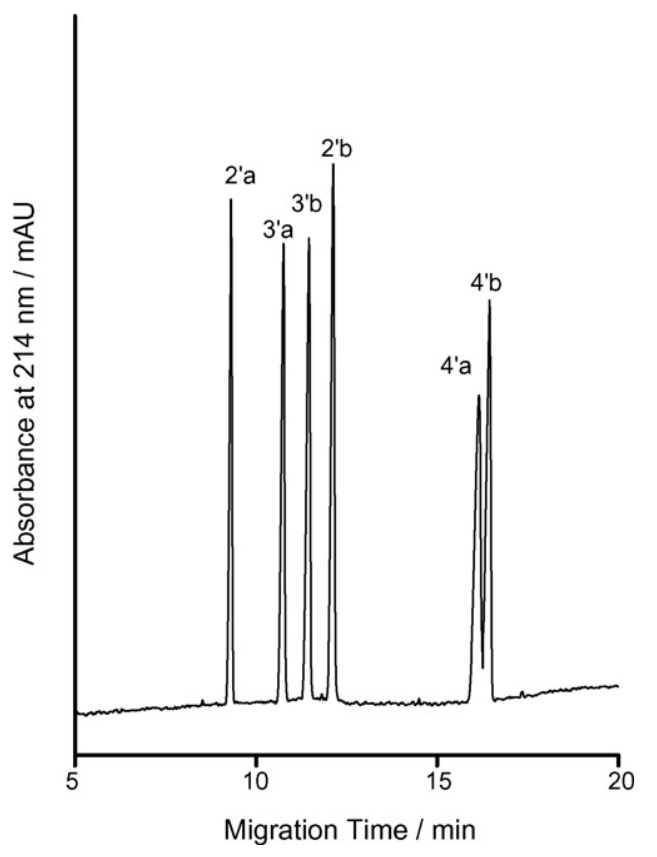

Fig. 5. Electropherogram of hydroxyflavanones obtained with dual CD systems consisting of $2.0 \%(\mathrm{w} / \mathrm{v}) \mathrm{S}-\beta-\mathrm{CD}$ and $2.0 \mathrm{mM} \gamma-\mathrm{CD}$ with the addition of $2.0 \mathrm{mM}$ SDS monomers in a phosphate buffer $(50 \mathrm{mM})$ at $\mathrm{pH} 3.0$. Other operating conditions are the same as for Fig. 2.

\subsubsection{Dual CD systems with the addition of SDS monomers}

To improve enantioseparation of hydroxyflavanones further, dual CD systems with the addition of SDS monomers as selectivity controller were attempted. Fig. 4 shows the electropherograms of three hydroxyflavanones obtained with dual CD system consisting of $2.0 \%(\mathrm{w} / \mathrm{v}) \mathrm{S}-\beta-\mathrm{CD}, 1.5 \mathrm{mM} \beta-\mathrm{CD}$ and $2.0 \mathrm{mM}$ SDS monomers, together with a CD system without the addition of $\beta$-CD in a phosphate buffer at $\mathrm{pH} 3.0$. As demonstrated, the enantioselectivity of hydroxyflavanones was found to enhance significantly with the use of a dual CD system. In fact, the enantioselectivities of $2^{\prime}$-hydroxyflavanone and $3^{\prime}$-hydroxyflavanone increased from 1.16 to 1.26 and from 1.03 to 1.06 , respectively. Moreover, the two enantiomeric peaks of $4^{\prime}$-hydroxyflavanone could be considerably resolved.

Fig. 5shows the electropherogram of hydroxyflavanones obtained with dual CD systems consisting of $2.0 \%$ (w/v) S- $\beta-C D$ and $2.0 \mathrm{mM} \gamma-\mathrm{CD}$ with $2.0 \mathrm{mM}$ SDS monomers in a phosphate buffer $(50 \mathrm{mM})$ at pH 3.0. Synergistic effect of dual CD systems on the enantioselectivity of hydroxyflavanone was demonstrated. The two enantiomeric peaks of 4 '-hydroxyflavanones were almost effectively separated. Thus, alternatively, similar effectiveness of the enantioseparation of hydroxyflavanones could be achieved with the use of a dual CD system. Table 2 illustrates the enhancement of the enantioselectivity of hydroxyflavanones with the use of dual CD systems in the presence of $2 \mathrm{mM}$ SDS monomers. The enantioselectivity of 2 -hydroxyflavanone increased from 1.16 to 1.33 , while the enantioselectivity of 3 '-hydroxyflavanone increased greatly from 1.03 to 1.07 . As the second enantiomeric peak of $3^{\prime}$-hydroxyflavanone was well separated from the second enantiomeric peak of 2 '-hydroxyflavanone, effective enantioseparation of hydroxyflavanones was better achieved with a dual CD system consisting of $S-\beta-C D$ and $\gamma-C D$ than with a dual CD system consisting of S- $\beta-C D$ and $\beta-C D$ in the presence of SDS monomers.

\subsection{Molecular structure vs. chiral recognition}

The basic molecular structures of $2^{\prime}$ - and $3^{\prime}$-hydroxyflavanone are similar to that of $4^{\prime}$-hydroxyflavanone. The crystal structure of $4^{\prime}$-hydroxyflavanone itself, as well as the $R$ - and $S$ enantiomer of $4^{\prime}$-hydroxyflavanone, has been described [47,48]. Flavanones adopt an envelope conformation [49]. The atom at $\mathrm{C}_{2}$ position is stickout from the $\mathrm{C}_{1} / \mathrm{C}_{3} / \mathrm{C}_{4} / \mathrm{C}_{5} / \mathrm{C}_{8}$ pyranone ring on one side for the $R$-enantiomer and on the other side from the pyranone ring for the $S$-enantiomer of $4^{\prime}$-hydroxyflavanone. For chiral recognition by CDs, the inclusion complex formation is usually required, although this is not a necessary prerequisite [50]. It has been shown that rutin, a natural flavone derivative, forms inclusion complex with $\beta$-CD [51,52]. The phenyl moiety of rutin was found to penetrate into the $\mathrm{CD}$ cavity to form a $1: 1$ inclusion complex. Accordingly, it is assumed that the complexes of hydroxyflavanones with S- $\beta$-CD may have a similar spatial configuration in the $\mathrm{CD}$ cavity as for rutin-CD complex. Thus hydrophobic interaction between the inner surface of the CD cavity and the phenyl moiety of hydroxyflavanone is considered to be one of the major forces responsible for binding. Actually, depending on the number and the substitution position of the hydroxyl group, the spatial configuration of an analyte in the CD cavity, as well as the penetration depth, may greatly affect chiral recognition.

In addition to inclusion complexation, hydrogen bonding interaction between hydroxyflavanones and S- $\beta-C D$ also play an important role in chiral recognition. There exists intermolecular hydrogen bonding between the hydroxyl groups at the outer rim of the CD cavity and the hydroxyl groups of hydroxyflavanone. It has been shown that the crystal packing of 5, 2'-dihydroxy-

Table 2

Enhancement of enantioselectivity of hydroxyflavanones with the use of dual CD systems in a phosphate buffer containing SDS monomers

\begin{tabular}{|c|c|c|c|}
\hline \multirow[t]{2}{*}{ CD systems } & \multicolumn{3}{|l|}{ Enantioselectivity } \\
\hline & 2'-Hydroxyflavanone & 3'-Hydroxyflavanone & $4^{\prime}$-Hydroxyflavanone \\
\hline$S-\beta-C D / S D S$ & 1.16 & 1.03 & 1.0 \\
\hline$S-\beta-C D / \gamma-C D / S D S$ & 1.33 & 1.07 & 1.04 \\
\hline
\end{tabular}

Dual CD systems consisting of S- $\beta-\mathrm{CD}(2.0 \%(\mathrm{w} / \mathrm{v}))$ and $\beta-\mathrm{CD}(1.5 \mathrm{mM})$ or $\gamma-\mathrm{CD}(2.0 \mathrm{mM})$ in a phosphate buffer at pH 3.0 containing $2.0 \mathrm{mM}$ SDS monomers. 
7-methoxyflavanone is stabilized by $\mathrm{O}-\mathrm{H} \cdots \mathrm{O}$ and $\mathrm{C}-\mathrm{H} \cdots \mathrm{O}$ hydrogen bonding [49]. Based on this argument, it is likely that an intramolecular hydrogen bonding between the $\mathrm{C}_{2}-\mathrm{H}_{2}$ bond and the $\mathrm{O}_{2^{\prime}}$ atom in 2'-hydroxyflavanone exists (Fig. 1B). Therefore, in correlation with the molecular structure, the higher chiral discrimination of $2^{\prime}$-hydroxyflavanone than the other two hydroxyflavanones is probably resulted from the spatial configuration of the $2^{\prime}$-hydroxyl substituent which may form an intramolecular hydrogen bonding around the stereogenic center and/or possess a steric repulsive interaction between the 2 '-hydroxyl substituent and the hydrophobic cavity of S- $\beta-C D$ [51]. Due to the involvement of multiple forces in analyte-CD complexes, analyte binding and chiral recognition mechanisms by CDs are not easily and clearly understandable.

\section{Conclusions}

S- $\beta-C D$ is an excellent chiral selector for enantioseparation of 2'-hydroxyflavanone. Enantioseparation of $3^{\prime}$ - and $4^{\prime}$-hydroxyflavanone aglycone can be effectively or nearly effectively achieved, respectively, with $S-\beta-C D$ alone or with dual $\mathrm{CD}$ systems consisting of $\mathrm{S}-\beta-\mathrm{CD}$ and a neutral $\mathrm{CD}$ with the addition of SDS monomers at low concentrations in a phosphate buffer at low $\mathrm{pH}$. Both inclusion complexation and hydrogen bonding interaction play important roles in chiral recognition of hydroxyflavanones. In correlation with the molecular structures, the higher chiral discrimination of 2 '-hydroxyflavanone than the other two hydroxyflavanones is probably resulted from the spatial configuration of the $2^{\prime}$-hydroxyl substituent which may form an intramolecular hydrogen bonding around the stereogenic center and/or possess a steric repulsive interaction between the $2^{\prime}$-hydroxyl substituent and the hydrophobic cavity of S- $\beta$-CD. Our preliminary results also show that the present approach is applicable to the enantioseparation of other hydroxyflavanone compounds.

\section{Acknowledgement}

We gratefully thank the National Science Council of Taiwan for financial support.

\section{References}

[1] E. de Rijke, P. Out, W.M.A. Niessen, F. Ariese, C. Gooijer, U.A.Th Brinkman, J. Chromatogr. A 1112 (2006) 31.

[2] C.A. Rice-Evans, N.J. Miller, G. Paganga, Trends Plant Sci. 2 (1997)152.

[3] W. Heller, G. Forkmann, in: J.B. Harborne (Ed.), The Flavonoids, Chapman \& Hall, London, 1988, p. 399.

[4] E. Wollenweber, in: V. Cody, E. Middleton Jr., J.B. Harborne, A. Beretz (Eds.), Plant Flavonoids in Biology and Medicine, II. Biochemical, Cellular and Medicinal Properties, Liss, New York, 1988, p. 45.

[5] K. Janssen, R.P. Mensink, F.J. Cox, J.L. Harryvan, R. Hovenier, P.C. Hollman, M.B. Katan, Am. J. Clin. Nutr. 67 (1998) 255.

[6] A.B. Jeppsson, U. Johansson, B. Waldeck, Acta Pharmacol. Toxicol. 54 (1984) 285

[7] G. Chen, H. Zhangb, J. Ye, Anal. Chim. Acta 423 (2000) 69.

[8] E. Dadakova, E. Prochazkova, M. Krizek, Electrophoresis 22 (2001)1573.

[9] C.W. Huck, G. Stecher, W. Ahrer, W.M. Sfoggl, W. Buchberger, G.K. Bonn, J. Sep. Sci. 25 (2002) 903.

[10] L. Suntornsuk, J. Pharm. Biomed. Anal. 27 (2002) 679.
[11] B.R. Baggett, J.D. Cooper, E.T. Hogan, J. Carper, N.L. Paiva, J.T. Smith, Electrophoresis 23 (2002) 1642.

[12] S.F. Wang, J.Y. Zhang, X.G. Chen, Z.D. Hu, Chromatographia 59 (2004) 507.

[13] R. Tsao, Z. Deng, J. Chromatogr. B 812 (2004) 85

[14] N. Volpi, Electrophoresis 25 (2004) 1872.

[15] L. Suntornsuk, O. Anurukvorakun, Electrophoresis 26 (2005) 648.

[16] A. Messina, C. Desiderio, A. De Rossi, F. Bachechi, M. Sinibaldi, Chromatographia 62 (2005) 409.

[17] C. Desiderio, A. De Rossi, M. Sinibaldi, J. Chromatogr. A 1081 (2005)99.

[18] X. Xu, X. Qi, W. Wang, G. Chen, J. Sep. Sci. 28 (2005) 647.

[19] S.P. Wang, K.J. Huang, J. Chromatogr. A 1032 (2004) 273.

[20] S. Bechmann, C.W. Huck, R. Bakry, G.K. Bonn, Electrophoresis 28 (2007) 799.

[21] N. Gel-Moreto, R. Streich, R. Galensa, J. Chromatogr. A 925 (2001) 279.

[22] N. Gel-Moreto, R. Streich, R. Galensa, Electrophoresis 24 (2003) 2716.

[23] Z. Aturki, M. Sinibaldi, J. Sep. Sci. 26 (2003) 844.

[24] D. Wistuba, O. Trapp, N. Gel-Moreto, R. Galensa, V. Schurig, Anal. Chem. 78 (2006) 3424

[25] M. Asztemborska, M. Mis' kiewicz, D. Sybilska, Electrophoresis 24 (2003) 2527.

[26] D. Wistuba, A. Bogdanski, K. Larsen, V. Schurig, Electrophoresis 27 (2006) 4359.

[27] J. Yáñez, P. Andrews, N. Davies, J. Chromatogr. B 848 (2007) 159.

[28] A.W. Lantz, R.V. Rozhkov, R.C. Larock, D.W. Armstrong, Electrophoresis 25 (2004) 2727.

[29] H. Park, S. Jung, Electrophoresis 26 (2005) 3833.

[30] A.M. Stalcup, K.H. Gahm, Anal. Chem. 68 (1996) 1360.

[31] K.H. Gahm, A.M. Stalcup, Chirality 8 (1996) 316.

[32] T. de Boer, R.A. de Zeeuw, G.J. de Jong, K. Ensing, Electrophoresis 21 (2000) 3220

[33] M. Fillet, Ph. Hubert, J. Crommen, J. Chromatogr. A 875 (2000)123.

[34] A.M. Abushoffa, M. Fillet, P. Hubert, J. Commen, J. Chromatogr. A 948 (2002) 321.

[35] M. Fillet, B. Chankvetadze, J. Crommen, G. Blaschke, Electrophoresis 20 (1999) 2691

[36] M. Fillet, L. Fotsing, J. Crommen, J. Chromatogr. A 817 (1998) 113

[37] F. Lelievre, P. Gareil, A. Jardy, Anal. Chem. 69 (1997) 385.

[38] K.H. Gahm, L.W. Chang, D.W. Armstrong, J. Chromatogr. A 759 (1997) 149.

[39] C. Danel, E. Lipka, J.P. Bonte, J.F. Goossens, C. Vaccher, C. Foulon, Electrophoresis 26 (2005) 3824.

[40] C.E. Lin, W.S. Liao, H.T. Cheng, C.M. Kuo, Y.C. Liu, Electrophoresis 26 (2005) 3869

[41] W.S. Liao, C.H. Lin, C.Y. Chen, C.M. Kuo, Y.C. Liu, J.C. Wu, C.E. Lin, Electrophoresis 28 (2007) 3922

[42] C.E. Lin, S.L. Lin, H.T. Cheng, I.J. Fang, C.M. Kuo, Y.C. Liu, Electrophoresis 26 (2005) 4187.

[43] W.M.Z. Wan Yunus, J. Taylor, D.M. Bloor, D.G. Hall, E. Wyn-Jones, J. Phys. Chem. 96 (1992) 8979.

[44] U.R. Dharmawardana, S.D. Christian, E.E. Tucker, R.W. Taylor, J.F. Scamehorn, Langmuir 9 (1993) 2258.

[45] J.W. Park, H.J. Song, J. Phys. Chem. 93 (1989) 6454

[46] C.E. Lin, H.C. Huang, H.W. Chen, J. Chromatogr. A 917 (2001) 297.

[47] M. Shoja, Z. Kristallogr, New Cryst. Struct. 213 (1998) 85.

[48] A. Biatonska, Z. Ciunik, E. Kostrzewa-Sustow, J. Dmochowska-Gtadysz, Acta Cryst. E63 (2007) 432

[49] M. Krishnaiah, R. Kumar, N. Kumar, D. Gunasekar, B. Jayaprakasam, Acta Cryst. E61 (2005) 3565.

[50] B. Chnakvetadze, Chem. Soc. Rev. 33 (2004) 337.

[51] M.L. Calabro, S. Tommasini, P. Dnato, R. Stancanelli, D. Raneri, S. Catania, C. Costa, V. Villari, P. Ficarra, R. Ficarra, J. Pharm. Biomed. Anal. 36 (2005) 1019.

[52] D. Haiyun, H. Ding, J. Chao, G. Zhang, S. Shung, J. Pan, Spectrochim. Acta A59 (2003) 3421. 